\title{
Indications for EMR/ESD in cases of early gastric cancer: relationship between histological type, depth of wall invasion, and lymph node metastasis
}

\author{
Shinji Ishikawa, Akihiko Togashi, Mituhiro Inoue, Shinobu Honda, Fumiaki Nozawa, Eitchirou Toyama, \\ Nobutomo Miyanari, Youichi Tabira, and Hideo Baba \\ Department of Gastroenterological Surgery, Graduate School of Medical Sciences, Kumamoto University, 1-1-1 Honjyo, Kumamoto, \\ Kumamoto 860-8556, Japan
}

\begin{abstract}
Background. Limited surgery by endoscopic mucosal resection (EMR) or endoscopic submucosal dissection (ESD) for gastric cancer is frequently performed in many institutions. These techniques do preserve gastric function and maintain a high quality of life but may compromise survival. The treatment strategy for early tumors should therefore be based on a complete cure, and limited surgery must thus have clear indications.

Methods. D2 gastric resection was performed in 278 early gastric adenocarcinomas, and a retrospective histological review of the specimens was made. The extended indications for EMR or ESD, according to the Japanese Gastric Cancer Association Treatment guidelines for gastric cancer in Japan, were also assessed.

Results. Of the 278 early gastric cancers, 115 were mucosal (M) cancers without ulcer. No lymph node metastases were seen in these specimens. Six of the 41 specimens of $M$ cancer with ulcers had lymph node metastases at the N1 level only. One of these had lymph node metastases from a tumor measuring less than $3 \mathrm{~cm}$ in size. Twenty-eight of 122 submucosal cancers had lymph node metastases $(23 \%)$. Twenty of these were SM1 tumors and 5 had lymph node metastases; 4 of these 5 had lymph node metastases despite the absence of vascular invasion.

Conclusion. Three cases had lymph node metastases that met the extended criteria for EMR/ESD. EMR and/or ESD should be limited to $M$ cancers without ulcer or differentiated-type $M$ cancer with ulcers smaller than $2 \mathrm{~cm}$. When the depth of tumor invasion is deeper than $M$, then a gastric resection with lymph node dissection is necessary.
\end{abstract}

Key words Early gastric cancer - Lymph node metastasis . $\mathrm{EMR} \cdot \mathrm{ESD}$

\section{Introduction}

The curability of early gastric cancer (EGC) depends on the lymph node dissection of metastatic lymph nodes. In most patients with EGC, lymph node metastasis does not exist, and limited surgery such as endoscopic mucosal resection (EMR) or endoscopic submucosal dissection (ESD) is now performed at many institutions [1,2]. Nevertheless, some patients do have lymph node metastasis and it is hard to detect cancer-positive lymph nodes by either endoscopic ultrasound (EUS) or computed tomography $(\mathrm{CT})[3,4]$. It is also difficult to diagnose such nodes during operation.

According to the Treatment guidelines for gastric cancer in Japan [5], the indications for EMR or ESD tend to be mucosal cancer within $2 \mathrm{~cm}$ in size, without ulcer. As many investigators are now trying to extend the indications, the guidelines have extended the indications for both EMR and ESD in the following cases; namely, (1) differentiated type, mucosal (M) cancer without ulcer, and larger than $2 \mathrm{~cm}$, (2) differentiated type, M cancer with ulcer, and $3 \mathrm{~cm}$ or smaller, and (3) undifferentiated type, $\mathrm{M}$ cancer without ulcer, and $2 \mathrm{~cm}$ or smaller [5]. In addition, the guidelines also state that an additional lymph node resection is not necessary when lymphovascular invasion is absent and when the tumor is not deeper than submucosal 1 (SM1; $500 \mu \mathrm{m})[5,6]$. Although these criteria may be enough to cure the majority of EGCs, as the target is "early" gastric cancer, the treatment strategy should nevertheless be based on a complete cure.

We herein report the relationship between lymph node metastasis and the depth of wall invasion of EGC. The result of this study shows the risk of lymph node metastasis of EGC and suggests that lymph node resection is still necessary for some EGC patients. 


\section{Patients and methods}

The records of 278 EGC patients at our institution from January 1980 to December 2004 were analyzed. All the EGCs were histologically proven adenocarcinoma of the stomach and were treated with D2 resection. The surgically removed lymph nodes were histologically examined for metastasis and the main tumors were sliced at intervals of $3 \mathrm{~mm}$ in order to determine the depth of invasion. All specimens were stained with hematoxylin and eosin and diagnosed by a histologist. Description of the cancer and histological evaluation of the resected specimens were performed in accordance with the Japanese classification of gastric carcinoma [7]. Lymph node metastasis-positive specimens were re-sliced from the middle (1.5-mm slice) and also re-evaluated by continuous sections to determine the depths of wall invasion and lymphovascular invasion.

The extended indications for EMR or ESD according to the Treatment guidelines for gastric cancer in Japan [5] are shown in Table 1 . The guidelines mention that treatment based on these extended indications has to be performed as a clinical trial. The resected gastric cancers were classified according to these criteria and then were evaluated for lymph node metastasis.

Table 1. Extended indications for EMR/ESD according to Treatment guidelines for gastric cancer in Japan [5]

(1) Differentiated type, mucosal cancer, UL (-), $>2 \mathrm{~cm}$

(2) Differentiated type, mucosal cancer, UL (+), $\leq 3 \mathrm{~cm}$

(3) Undifferentiated type, mucosal cancer, UL (-), $\leq 2 \mathrm{~cm}$

Additional lymph node resection is not necessary when lymphovascular invasion is absent $(\mathrm{ly} 0, \mathrm{v} 0)$ and also when it is not deeper than SM1 $(\sim 500 \mu \mathrm{m})$

UL, ulcer; ly, lymph duct invasion; v, vascular invasion

\section{Results}

The clinicopathological characteristics of the 278 EGCs are shown in Table 2. A total of $156 \mathrm{M}$ cancers and 122 SM cancers were analyzed. Of the M cancers, 115 were without ulcer and had no lymph node metastasis, and 41 were cancers with ulcer, with lymph node metastases in $6(14.6 \%)$. It is interesting to note that these 6 EGCs had lymph node metastases only at the N1 level. Of the SM cancers, 101 were without ulcers, but there were lymph node metastases in $18(17.8 \%)$. The 21 remaining SM cancers had ulcers, and 10 of these had lymph node metastases (47.6\%).

Figure 1 shows the details of the $156 \mathrm{M}$ cancers. Of the $41 \mathrm{M}$ cancers with ulcers, 32 were differentiated type and 4 had lymph node metastasis. Twenty-one tumors were $3 \mathrm{~cm}$ or smaller, and of these, 1 had lymph node metastasis, which thus met the criteria for the extended indications of EMR or ESD.

Table 2. Clinicopathological characteristics of 278 early gastric cancers

\begin{tabular}{lcc}
\hline & Mucosal (M) & Submucosal (SM) \\
\hline Number & 156 & 122 \\
Age, in years (mean) & 62.1 & 62.3 \\
Sex & & \\
$\quad$ Male:Female & $2.0: 1$ & $2.6: 1$ \\
Ulcer & & \\
$\quad(-)$ & 115 & 101 \\
$\quad(+)$ & 41 & 21 \\
Histology & & \\
$\quad$ Differentiated & 90 & 77 \\
$\quad$ Undifferentiated & 66 & 45 \\
Lymph node metastasis & 150 & 94 \\
$\quad(-)$ & 6 & 28 \\
$\quad(+)$ & & \\
\hline
\end{tabular}

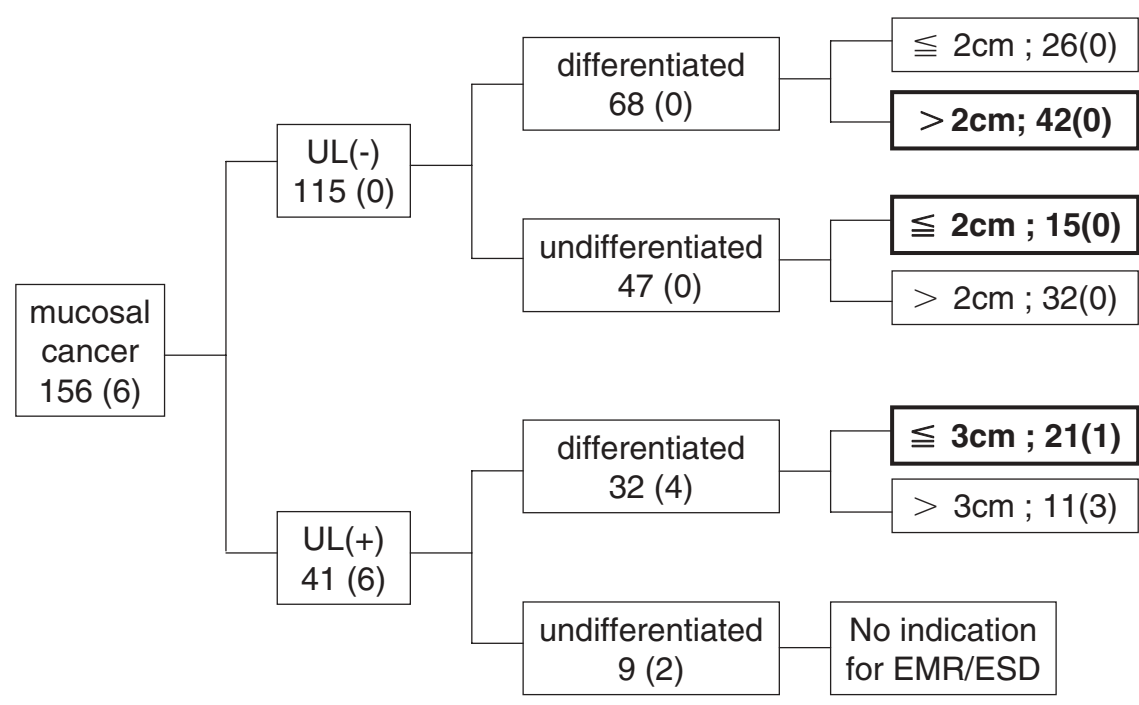

Fig. 1. Determination of mucosal cancer; 156 mucosal cancer cases were evaluated according to the existence of ulcer, histological type, size, and lymph node metastasis. The extended criteria for endoscopic mucosal resection $(E M R)$ or endoscopic submucosal dissection (ESD) are indicated in bold type. Numbers in parentheses show number of cases with lymph node metastasis. $U L$, ulcer 


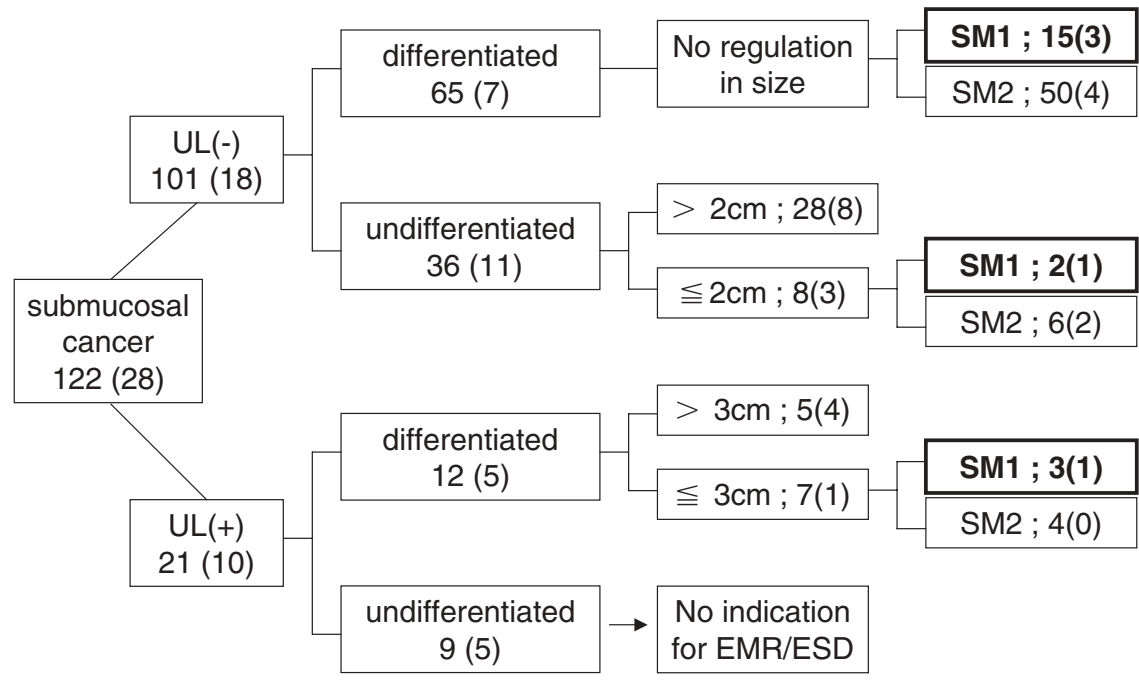

Fig. 2. Determination of submucosal cancer; 122 submucosal cancer cases were evaluated according to the existence of ulcer, histological type, size, depth of wall invasion, and lymph node metastasis. The extended criteria for EMR or ESD are indicated in bold type. Numbers in parentheses show number of cases of lymph node metastasis. UL, ulcer; SM1, SM2, submucosal layers classified according to the Japanese classification of gastric carcinoma, 13th edition [7]

Table 3. Lymph node metastases cases that met extended indication criteria for EMR/ ESD

\begin{tabular}{lcccccc}
\hline Histology & Type & Ulcer & Depth & Size & N & $\begin{array}{c}\text { Lymphovascular } \\
\text { invasion }\end{array}$ \\
\hline 1. Differentiated & IIc + III $^{\mathrm{a}}$ & + & M & $2.0 \times 2.0$ & 1 & ly0, v0 \\
2. Differentiated & IIc $^{\mathrm{b}}$ & - & SM1 & $2.0 \times 0.8$ & 2 & ly0, v0 \\
3. Differentiated & IIc + III & + & SM1 & $1.9 \times 1.3$ & 2 & ly0, v0 \\
\hline
\end{tabular}

$\mathrm{N}$, lymph node level; ly, lymph duct invasion; $\mathrm{v}$, vascular invasion

${ }^{a}$ Depressed type with ulcer

${ }^{\mathrm{b}}$ Depressed type

The details of the $122 \mathrm{SM}$ cancers are shown in Fig. 2. There were 65 differentiated SM cancers without ulcer, and 15 of these 65 were SM1, with 3 of the 15 having lymph node metastasis. Lymphovascular invasion was absent in 2 of these 3 SM1 cancers with lymph node metastasis. Eight SM cancers of undifferentiated type were without ulcers and were $2 \mathrm{~cm}$ or smaller. Of these 8, 2 were SM1 and 1 of these 2 had lymph node metastasis despite there being no lymphovascular invasion. A total of $12 \mathrm{SM}$ cancers were differentiated type with ulcers. Seven of these 12 differentiated SM cancers were $3 \mathrm{~cm}$ or smaller and 1 of the 7 was SM1 without lymphovascular invasion.

Of the 278 patients with EGC, 3 had lymph node metastasis, which met the extended indication criteria for EMR or ESD. The characteristics of these 3 cases are shown in Table 3. Patient 1 developed bone metastasis and died 4 years after surgery, and the other two patients are still alive. One patient had $\mathrm{M}$ cancer and the other 2 had SM1 cancer. The M cancer was differentiated type with ulcer and it measured $2 \mathrm{~cm}$ in diameter.

\section{Discussion}

The major prognostic factor in early gastric cancer (EGC) is lymph node metastasis [8,9]. Because most EGCs do not have lymph node metastasis, and limited surgery not only preserves stomach function but can also help to maintain the patient's quality of life (QOL), many institutions select EMR or ESD as the treatment method for EGC. But some patients definitely have lymph node metastasis, and as the target is "early," the treatment strategy has to be based on the goal of obtaining a complete cure. The detection of metastatic lymph nodes by endoscopic ultrasound (EUS) or computed tomography $(\mathrm{CT})$ is not effective $[3,4]$. Also, a correct diagnosis of metastases is impossible during surgery. This means that if lymph node metastasis exists at the time of EMR or ESD, and an additional lymph node resection is not added, such an unresected metastatic lymph node will grow and eventually result in recurrence.

The data from this study showed that in 278 EGCs, lymph node metastasis was observed in 3 cases that met the extended indication criteria. As the main tumor and the surrounding normal tissues were sliced at intervals of $1.5 \mathrm{~mm}$ and also evaluated by continuous section, 
diagnosis of the depth of wall invasion must have been accurate. For determining lymphovascular invasion, a histological examination was done only by hematoxylin and eosin staining and no immunohistochemistry was performed. There is a possibility that examinations using vascular endothelial growth factor (VEGF) or D2-40 may influence the diagnosis of lymphovascular invasion [10-12]. However, histological examination is generally done by hematoxylin and eosin staining. We therefore concluded that the evaluation of lymphovascular invasion should be performed only by hematoxylin and eosin staining.

Our study showed that mucosal (M) cancer without ulcers had no lymph node metastasis, thus suggesting that $\mathrm{M}$ cancer without ulcers may be a good indication for EMR or ESD regardless of the size or histological type. For extended indication criterion no. 2 of the Treatment guidelines for gastric cancer in Japan [5] (differentiated type, $\mathrm{M}$ cancer with ulcer and $3 \mathrm{~cm}$ or smaller), 1/21 (4.7\%) had lymph node metastases (Table 3 ; case 1). In regard to the size of $M$ cancer with ulcer, there was no lymph node metastasis when the size was smaller than $2 \mathrm{~cm}$, thus indicating that $\mathrm{M}$ cancer smaller than $2 \mathrm{~cm}$ with an ulcer might be an indication for EMR or ESD. Although the patient noted above (case 1 in Table 3) was operated on, the outcome was death due to bone metastasis. Therefore, even operation cannot result in complete cure in such cases. When the depth of wall invasion was SM1, we found that 2/15 (13\%) of EGC differentiated type without ulcer had lymph node metastases, $1 / 2(50 \%)$ of undifferentiated type without ulcer had lymph node metastasis, and 1/3 (33\%) of differentiated type with ulcer had lymph node metastasis. Of these four SM1 EGCs, two met the extended indication criteria (Table 3, cases 2 and 3). The details of the other two SM1 EGCs were: SM1 cancer, differentiated type, without ulcer, $5.4 \times 3.5 \mathrm{~cm}$, ly0, v0; and SM1 cancer, undifferentiated type, without ulcer, $2.0 \times 2.0 \mathrm{~cm}$, ly 0 , v0. Although the guidelines are not clear in this regard, according to the data published in the guidelines, the lymph node metastasis-negative criteria for SM1 cancer are "differentiated type, smaller than $3 \mathrm{~cm}$ " [5]. For this reason we excluded these two cases from the extended indication criteria. However, if these cases had been first diagnosed as $\mathrm{M}$ cancer clinically, investigators would have performed EMR/ESD for certain. The question is, what would happen if the results of histological examination after EMR/ESD were to show findings similar to these two cases above?
According to the data we, as well as others, have shown that at present there is no accurate tool to detect lymph node metastasis, except for dissection. We therefore concluded that the indications for EMR or ESD should be limited to mucosal cancer without ulcers or to differentiated-type mucosal cancer smaller than $2 \mathrm{~cm}$ with ulcers. In conclusion, even when invasion is shallow, submucosal cancer nevertheless requires additional lymph node dissection.

\section{References}

1. Ono H. Endoscopic submucosal dissection for early gastric cancer. Chin J Dig Dis 2005;6:119-21.

2. Furukawa $H$, Imamura $H$, Kodera $Y$. The role of surgery in the current treatment of gastric carcinoma. Gastric Cancer 2002;5: 13-6.

3. Tsendsuren T, Jun SM, Mian XH. Usefulness of endoscopic ultrasonography in preoperative TNM staging of gastric cancer. World J Gastroenterol 2006;12:43-7.

4. Polkowski M, Palucki J, Wronska E, Szawlowski A, NasierowskaGuttmejer A, Butruk E. Endosonography versus helical computed tomography for locoregional staging of gastric cancer. Endoscopy 2004;36:617-23.

5. Japanese Gastric Cancer Association. Treatment guidelines for gastric cancer in Japan (in Japanese). 2nd ed. Tokyo: Kanehara; 2004.

6. Abe N, Sugiyama M, Masaki T, Ueki H, Yanagida O, Mori T, et al. Predictive factors for lymph node metastasis of differentiated submucosally invasive gastric cancer. Gastrointest Endosc 2004; 60:242-5.

7. Japanese Gastric Cancer Association. Japanese classification of gastric carcinoma (in Japanese). 13th ed. Tokyo: Kanehara; 1999.

8. Yokota T, Ishiyama S, Saito T, Teshima S, Narushima Y, Murata $\mathrm{K}$, et al. Lymph node metastasis as a significant prognostic factor in gastric cancer: a multiple logistic regression analysis. Scand $\mathbf{J}$ Gastroenterol 2004;39:380-4.

9. Skoropad V, Berdov B, Zagrebin V. Clinicopathological features and outcome of surgical treatment of 149 patients with early (pT1) gastric cancer. Onkologie 2005;28:247-52.

10. Juttner S, Wissmann C, Jons T, Vieth M, Hertel J, Gretschel S, et al. Vascular endothelial growth factor-D and its receptor VEGFR-3: two novel independent prognostic markers in gastric adenocarcinoma. J Clin Oncol 2006;24:228-40.

11. Shida A, Fujioka S, Kobayashi K, Ishibashi Y, Nimura H, Mitsumori N, et al. Expression of vascular endothelial growth factor (VEGF)-C and -D in gastric carcinoma. Int J Clin Oncol 2006;11:38-43.

12. Arigami T, Natsugoe S, Uenosono Y, Arima H, Mataki Y, Ehi $\mathrm{K}$, et al. Lymphatic invasion using D2-40 monoclonal antibody and its relationship to lymph node micrometastasis in $\mathrm{pN} 0$ gastric cancer. Br J Cancer 2005;93:688-93. 